\begin{tabular}{|c|l|}
\hline Title & $\begin{array}{l}\text { Progressive left ventricular dysfunction and my ocardial fibrosis in Duchenne and Becker muscular dystrophy : a } \\
\text { longitudinal cardiovascular magnetic resonance study }\end{array}$ \\
\hline Author(s) & $\begin{array}{l}\text { Aikawa, T adao; Takeda, A tsuhito; Oyamar Manabe, Noriko; Naya, Masanao; Y amazawa, Hirokuni; Koy anagawa, } \\
\text { Kazuhiro; Ito, Yoichi M.; A nzai, Toshihisa }\end{array}$ \\
\hline Citation & $\begin{array}{l}\text { Pediatric Cardiology, 40(2), 384-392 } \\
\text { https://doi.org/10.1007/300246-018-2046-x }\end{array}$ \\
\hline Issue Date & 2019-02 \\
\hline Doc URL & http://hdl.handle.net/2115/76647 \\
\hline Rights & The final publication is available at link.springer.com \\
\hline Type & article (author version) \\
\hline File Information & HUSCAP_PEDC-D-18-00579.pdf \\
\hline
\end{tabular}

Instructions for use 


\title{
Progressive left ventricular dysfunction and myocardial fibrosis in Duchenne and Becker muscular dystrophy: a longitudinal cardiovascular magnetic resonance study
}

\author{
Tadao Aikawa ${ }^{1}$, Atsuhito Takeda ${ }^{2}$, Noriko Oyama-Manabe ${ }^{3 *}$, Masanao Naya ${ }^{1}$, Hirokuni \\ Yamazawa $^{2}$, Kazuhiro Koyanagawa ${ }^{1}$, Yoichi M. Ito ${ }^{4}$, Toshihisa Anzai ${ }^{1}$
}

${ }^{1}$ Department of Cardiovascular Medicine, Hokkaido University Hospital, Kita-14, Nishi-5, Kita-ku, Sapporo 060-8648, Japan; ${ }^{2}$ Department of Pediatrics, Hokkaido University Hospital, Kita-14, Nishi-5, Kita-ku, Sapporo 060-8648, Japan; ${ }^{3}$ Department of Diagnostic and Interventional Radiology, Hokkaido University Hospital, Kita-14, Nishi-5, Kita-ku, Sapporo 060-8648, Japan; ${ }^{4}$ Department of Biostatistics, Faculty of Medicine and Graduate School of Medicine, Hokkaido University, Kita-15, Nishi-7, Kita-ku, Sapporo 060-8638, Japan.

\section{Address for Correspondence:}

Noriko Oyama-Manabe, $\mathrm{MD}, \mathrm{PhD}$

Department of Diagnostic and Interventional Radiology

Hokkaido University Hospital

Kita-14, Nishi-5, Kita-ku, Sapporo 060-8648, Japan

Tel.: +81-11-706-7779

Fax: +81-11-706-7876

E-mail: norikooyama@med.hokudai.ac.jp

\section{Acknowledgements}

We thank Drs. Yukitoshi Isikawa and Yuka Ishikawa for providing DMD/BMD specific knowledge and insight and Kinya Ishizaka for technical assistance. This study was funded by the Miyata Cardiac Research Promotion Foundation (to T.A.). The funder had no role in the study design, data collection, analysis, interpretation of data, or in the writing of the manuscript. 


\section{Abstract}

This study examined the progression of left ventricular dysfunction and myocardial fibrosis in patients with Duchenne muscular dystrophy (DMD) or Becker muscular dystrophy (BMD) to evaluate the effects of angiotensin-converting enzyme inhibitor (ACEI). Ninety-eight cardiovascular magnetic resonance (CMR) studies in 34 consecutive patients with DMD ( $n=21)$ or BMD ( $n=13)$ were retrospectively reviewed. Left ventricular ejection fraction (LVEF) and the extent of myocardial late gadolinium enhancement (LGE) were semiautomatically quantified. During the study period, 5 patients had already been treated with ACEI at the first CMR; 5 were started on ACEI at LVEF $\geq 55 \%$ and 10 at $\mathrm{LVEF}<55 \%$. All patients had hyperenhanced myocardium on LGE images at the first CMR (median extent, 3.3\%; interquartile range, 0.1\%-14.3\%). A mixed-effects model for longitudinal data of each patient, adjusted for age, type of muscular dystrophy, steroid use, and ACEI use, showed that higher age $(\beta=-1.1 \% /$ year; $95 \%$ confidence interval $[\mathrm{CI}],-1.8 \%$ to $-0.4 \%$; $p=0.005)$ and no use of $\operatorname{ACEI}(\beta=-3.1 \% ; 95 \% \mathrm{CI} ;-5.4 \%$ to $-0.8 \% ; p=0.009)$ were significantly associated with a lower LVEF. When ACEI use was stratified by time of initiation ( $\mathrm{LVEF} \geq 55 \%$ vs. $<55 \%$ ), only ACEI initiation at $\mathrm{LVEF}<55 \%$ had a beneficial effect on LVEF at each imaging examination ( $\beta=3.7 \%$; $95 \%$ CI, $0.9 \%$ to $6.4 \% ; p=0.010$ ). ACEI use or the time of initiation of ACEI did not significantly affect age-related increase in LGE.

Conclusion: ACEI attenuated the age-related decline in LVEF only in patients with DMD or BMD and reduced LVEF, suggesting that further investigation on prophylactic use of cardioprotective therapy in these patients is warranted.

Keywords: Duchenne muscular dystrophy; Becker muscular dystrophy; Angiotensin-converting enzyme inhibitor; Cardiovascular magnetic resonance imaging; Late gadolinium enhancement; Mixed-effects model. 


\section{Introduction}

Duchenne muscular dystrophy (DMD) and Becker muscular dystrophy (BMD) are X-linked disorders caused by mutations in the dystrophin gene and characterized by progressive wasting and weakness of skeletal muscles. Although survival in patients with DMD has improved with advances in respiratory care, cardiac complications remain an important determinant of prognosis in patients with DMD [1]. BMD is a milder and more variable phenotype, which maintains the open reading frame of dystrophin; nevertheless, cardiac involvement is seen in approximately $70 \%$ of patients with BMD by the age of 40 years [2]. The most common form of cardiac involvement in patients with DMD or BMD is dilated cardiomyopathy [3, 4], presenting as an age-related progressive decline in left ventricular ejection fraction (LVEF) accompanied by myocardial fibrosis, which is detected by late gadolinium enhancement (LGE) cardiovascular magnetic resonance (CMR) imaging [5, 6]. To delay the progression of dilated cardiomyopathy in DMD and BMD, the American Heart Association scientific statement recommends the use of an angiotensin-converting enzyme inhibitor (ACEI) or angiotensin receptor blocker in patients with reduced LVEF [7]. Furthermore, previous studies reported that the prophylactic use of ACEI in patients with DMD or BMD and normal LVEF was associated with delaying myocardial fibrosis [8] and decreasing all-cause mortality [9]. However, the prophylactic effects of ACEI on LVEF and the extent of LGE in these patients have not been fully investigated. Therefore, we examined the progression of left ventricular dysfunction and myocardial fibrosis using CMR in DMD and BMD cohorts to evaluate the longitudinal effects of ACEI on LVEF and the extent of LGE. 


\section{Material and methods}

\section{Study population}

All consecutive patients with DMD ( $n=21)$ or BMD ( $n=13)$ referred for clinically indicated CMR to our hospital between January 2013 and August 2017 were retrospectively included in this study. Patients with other neuromuscular disease were excluded. The study was approved by the Institutional Review Board (IRB No. 017-0207) with a waiver of written informed consent. The medical records of all patients in the study were reviewed, and DMD or BMD was ascertained by a clinical examination, dystrophin gene analysis by multiplex ligation-dependent probe amplification, the absence or reduction of dystrophin in muscle biopsy, and family history of DMD or BMD. Every clinical visit consisted of a review of medication use and blood tests, including total creatine kinase, brain natriuretic peptide (BNP), human atrial natriuretic peptide, and troponin T. According to the current recommendations [7], ACEI treatment was started in patients with reduced LVEF basically, although the time of ACEI initiation was considered on an individual basis at discretion of their physicians.

\section{CMR imaging protocol}

CMR imaging was performed using a 1.5-T whole-body scanner (Achieva; Philips Medical Systems, Best, the Netherlands) with a 5-channel phased-array cardiac coil during repeated breath-holds if tolerated. After localization of the heart, left ventricular (LV) short-axis cine images were obtained using a retrospectively electrocardiogram-gated, balanced steady-state free precession pulse sequence with the following parameters: field of view, $380 \mathrm{~mm}$; repetition time, $2.8 \mathrm{~ms}$; echo time, $1.38 \mathrm{~ms}$; flip angle, $60^{\circ}$; slice thickness, 10 mm; acquisition matrix, $192 \times 164$ pixels; sensitivity encoding parallel imaging factor, 2.5; and 20 phases per cardiac cycle. LGE images were obtained 10-15 min after administration 
of $0.1 \mathrm{mmol} / \mathrm{kg}$ of Gd-DTPA (Magnevist; Bayer, Osaka, Japan) using an inversion-recovery prepared, 3-dimensional fast field echo pulse sequence with the following parameters: spectral presaturation with inversion recovery for fat suppression; field of view, $400 \mathrm{~mm}$; repetition time, $3.4 \mathrm{~ms}$; echo time, $1.04 \mathrm{~ms}$; flip angle, $15^{\circ}$; slice thickness, $5 \mathrm{~mm}$ with no gap; inversion time, 250-290 ms; acquisition matrix, $224 \times 96$ pixels; and sensitivity encoding parallel imaging factor, 2. The optimal inversion time was selected to null the signal from normal myocardium using a Look-Locker sequence. Sedation during CMR was performed at the physician's discretion. Body weight and height were ascertained at the time of CMR examination by interviewing patients and their parents or by using medical records. Body surface area was calculated using the Dubois formula: body weight $(\mathrm{kg})^{0.425} \times$ height $(\mathrm{cm})^{0.725} \times 0.007184$.

\section{CMR image analysis}

CMR image analysis was performed using commercially available software (Ziostation2; Ziosoft Inc., Tokyo, Japan). LV volumes, mass, and LVEF were measured by semiautomatically outlining endocardial and epicardial borders of the myocardium on the short-axis cine images, as previously described [10]. The LV myocardium was automatically divided into 16 segments of the American Heart Association 17-segment model, excluding the apex segment. Two observers (with 4 and 20 years of experience in cardiac imaging, respectively) independently assessed the presence of hyperenhanced myocardium in each segment on LGE images. After the initial assessment, any interobserver disagreements were resolved by consensus. The extent of hyperenhanced myocardium on LGE images was quantified as the percentage area of hyperenhanced myocardium using a 5-standard deviation threshold above the mean of remote myocardium $[8,11]$ (Fig. 1). We defined substantial LGE as the extent of hyperenhancement $\geq 5 \%$ of 
total LV mass on the basis of previous study [12].

\section{Statistical analysis}

Continuous variables are presented as medians with interquartile range (IQR). Categorical variables are presented as absolute numbers with percentages. Differences between groups were evaluated using the Wilcoxon rank sum test for continuous data and the Fisher exact test for categorical data. Correlations between continuous variables were examined using Spearman rank correlation coefficients $(\rho)$. We assessed the relationship between age-related decline in LVEF or increase in the extent of LGE, the type of muscular dystrophy, and medication use (steroid and ACEI) using a mixed-effects model with a random intercept and slope for longitudinal data of each patient [6] to maximize statistical power. To test the effectiveness of prophylactic use of ACEI, a stratified analysis by time of initiation of the ACEI (initiated at LVEF $\geq 55 \%$ vs. $<55 \%$ ) was performed. $\beta$-blocker use was not adjusted in the mixed-effects model analysis [13] because a previous study reported that concomitant use of $\beta$-blockers with ACEI did not affect LVEF in patients with DMD [14]. A 2-tailed $p$ value of $<0.05$ was considered statistically significant. All statistical analyses were performed using JMP Pro 13.1.0 (SAS Institute Inc., Cary, NC). 


\section{Results}

\section{Patient characteristics}

In total, 34 patients underwent at least 1 CMR study during the study period (total 98 studies), and 24 of these patients had $\geq 2$ CMR studies (median follow-up duration, 3.0 years; range, 0.6-4.1 years). During the follow-up period, none of the patients died or was admitted for decompensated heart failure. Baseline patient characteristics at the first and last CMR are summarized in Table 1 and 2, respectively. At the initial examination, the median patient age was 11.9 years (IQR, 6.4-15.9 years) and 33 patients (97\%) were men. Among the 21 patients with DMD, 8 (38\%) were already wheelchair-bound and 8 (38\%) were being treated with prednisolone (median dose, $7.0 \mathrm{mg} /$ day; IQR, 5.0-8.3 mg/day). Five patients with DMD (median LVEF, 37.5\%; IQR, 22.6\%-58.0\%) were being treated with ACEI, and 3 of these 5 patients who had an LVEF $<55 \%$ were also being treated with $\beta$-blocker. During the study period, 5 patients were started on ACEI at LVEF $\geq 55 \% ; 10$ at LVEF $<55 \%$; and none of the patients died. Among patients treated with ACEI $(n=20), 1$ patient was prescribed cilazapril (1.5 mg/day), and the other patients were prescribed enalapril, of which median dose achieved at the end of the study period was $10 \mathrm{mg} /$ day (IQR, 7.2-10.0 mg/day). All patients treated with $\beta$-blockers $(n=5)$ were prescribed bisoprolol, of which median dose achieved at the end of the study period was $2.5 \mathrm{mg} / \mathrm{day}$ (IQR, 0.625-2.5 mg/day). At baseline, 9 patients had elevated BNP levels (>18.4 pg/mL) and 25 had elevated serum troponin T levels $(>0.014 \mathrm{ng} / \mathrm{mL})$. The levels of serum total creatine kinase, troponin T, and plasma BNP in patients with DMD were significantly higher than those in patients with BMD (Table 1).

\section{Baseline CMR imaging results and relationship with clinical variables}

Baseline CMR results were similar in patients with DMD and BMD (Table 1); there was no 
significant difference in baseline LVEF and end-diastolic and end-systolic volume indices. One patient with DMD underwent only cine CMR and did not undergo LGE imaging at the time of the first CMR study because he could not lie still in bed during the examination. All other patients were considered as LGE positive on visual assessment, which was typically the subepicardial location in the lateral wall. The extent of LGE at the first CMR was varied (median extent, 3.3\% of LV mass; IQR, 0.1\%-14.3\%). Patients with substantial LGE ( $\geq 5 \%$ of total LV mass) ( $n=14)$ had a lower LVEF than those without $(n=19)(42.7 \%$ [IQR, $32.3 \%-58.2 \%$ ] vs. $55.1 \%$ [IQR, 52.6\%-58.5\%], $p=0.040$ ). None of the patients aged $<10$ years $(n=14)$ had substantial LGE, and 8 patients (6 DMD and 2 BMD) aged $<10$ years had LVEF $<55 \%$.

Regarding the first CMR, the extent of LGE showed a negative correlation with LVEF (Spearman $\rho=-0.59, p<0.001$ ) (Fig. 2), end-diastolic volume index (Spearman $\rho=0.56$, $p<0.001$ ), and end-systolic volume index (Spearman $\rho=0.62, p<0.001)$. Among CMR variables and blood test data, the levels of plasma BNP were significantly correlated with LVEF (Spearman $\rho=-0.56, p<0.001$ ) and the extent of LGE (Spearman $\rho=0.64, p<0.001$ ). The levels of serum troponin $\mathrm{T}$ and plasma human atrial natriuretic peptide were not significantly correlated with any CMR variables. The elevated levels of plasma BNP for detecting substantial LGE, with a cutoff value of $\geq 18.4 \mathrm{pg} / \mathrm{mL}$, yielded a sensitivity of $57 \%$, a specificity of $95 \%$, a positive predictive value of $89 \%$, and a negative predictive value of 75\%. The area under the curve of plasma BNP for detecting substantial LGE was 0.79 .

\section{Longitudinal relationship between age and LVEF in muscular dystrophy}

A mixed-effects model including only age showed that LVEF significantly decreased by 0.7\% per year (95\% confidence interval [CI], 0.004\%-1.3\%; $p=0.049$ ) (Fig. 3). On including the type of muscular dystrophy and a linear interaction term (age $\times$ BMD) in this 
model, LVEF significantly decreased by $1.1 \%$ per year (95\% CI, $0.2 \%-1.9 \%$; $p=0.016$ ), whereas additional variables were not significantly associated with LVEF ( $p=0.78$ and $p=0.20$, respectively), indicating that the type of muscular dystrophy did not attenuate age-related decline in LVEF.

\section{Longitudinal effect of ACEI on LVEF in muscular dystrophy}

Table 3 summarizes the results of mixed-effects models including medication use to identify predictors of LVEF in patients with DMD or BMD. In a multivariate analysis including age, the type of muscular dystrophy, and medication use (Model 1), lower age and ACEI use were significantly associated with a higher LVEF ( $p=0.005$ and $p=0.009$, respectively). When the time of initiation of ACEI was stratified (Model 2), lower age and ACEI initiation at LVEF $<55 \%$ were significantly associated with a higher LVEF $(p=0.005$ and $p=0.010$, respectively), whereas ACEI introduction at LVEF $\geq 55 \%$ was not significantly associated with a higher $\operatorname{LVEF}(p=0.24)$.

\section{Longitudinal relationship between age, treatment, and myocardial fibrosis in muscular dystrophy}

Table 4 summarizes the results of mixed-effects models to identify predictors of the extent of LGE in patients with DMD or BMD. In a multivariate analysis including age and the type of muscular dystrophy (Model 1), a higher age was significantly associated with a larger extent of LGE ( $p=0.005$ ). ACEI use (Model 2) or the time of initiation of ACEI (Model 3) did not significantly affect age-related increase in LGE. 


\section{Discussion}

This study showed that ACEI initiation at LVEF $<55 \%$, but not LVEF $\geq 55 \%$, significantly attenuated the age-related decline in LVEF in patients with DMD or BMD. However, ACEI use did not affect the age-related increase in myocardial fibrosis detected by LGE. Importantly, ACEI treatment for DMD and BMD may be effective only in patients with reduced LVEF.

ACEI are a standard therapy for DMD and BMD with systolic heart failure [7]. It was found that ACEI initiation at LVEF $<55 \%$ was significantly associated with a higher LVEF in patients with DMD or BMD, which is in line with previous reports [3, 13]. We also found that there was no significant association between ACEI initiation at LVEF $\geq 55 \%$ and progressive decline in LVEF. In 2005, Duboc et al. reported on the prophylactic effects of ACEI on LVEF in patients with DMD with LVEF > 55\% (mean LVEF, 65.0\% $\pm 5.4 \%$ ) [15]. However, this earlier randomized trial did not show a significant difference in mean LVEF between patients treated with and without ACEI at 3-year follow-up (60.7\% $\pm 7.6 \%$ vs. $64.4 \% \pm 9.8 \% ; p=$ not significant). Although they also reported that the prophylactic use of ACEI was significantly associated with a lower mortality in patients with DMD after 10-year follow-up [9], the effects of steroid therapy, which yielded a survival benefit for patients with DMD [16], were not considered in these results. Recently, Silva et al. reported that in their randomized clinical trial, early initiation of ACEI was associated with an attenuation of myocardial fibrosis progression in patients with DMD or BMD with LVEF $\geq 50 \%$ [8]; however, they also reported that there was no significant difference in 2-year decline in LVEF between patients treated with and without ACEI $(-6.2 \% \pm 6.0 \%$ vs. $-4.9 \%$ $\pm 6.4 \%, p=0.94)$. Although ACEI treatment is usually well tolerated, it is well known that use of ACEI can cause chronic cough, symptomatic hypotension and angioedema. Given the lack of conclusive evidence on the benefit of prophylactic ACEI for DMD or BMD patients 
with normal LVEF, we should be careful to ensure that ACEI treatment is effective in all stages of the disease. These results also suggest that annual CMR is preferable for patients with normal LVEF to detect changes in LVEF and myocardial fibrosis.

ACEI use or the time of initiation of ACEI did not significantly affect age-related progressive myocardial fibrosis detected by LGE. On the basis of this finding, only a small fraction of the benefits of ACEI could be attributed to a reduction in myocardial fibrosis, suggesting that the benefits of ACEI among patients with reduced LVEF is mediated by reduction in left ventricular preload and afterload or reduction in sympathetic stimulation [17].

The extent of LGE was moderately correlated with LVEF and LV volume. However, we found that 8 patients aged $<10$ years had an LVEF $<55 \%$ even without substantial LGE ( $\geq 5 \%$ of total LV mass). Although LGE is well established for detecting myocardial fibrosis, these findings suggest that LGE may not always detect fibrotic changes, particularly when these occur diffusely in patients with DMD or BMD. This concern has been raised about a variety of other nonischemic cardiomyopathies [18]. In this regard, previous studies suggested that circumferential strain analysis [19] or T1 and extracellular volume fraction mapping [20] using CMR may be more suited to detecting diffuse myocardial damage in patients with DMD and normal LVEF.

Cardiac biomarkers for early detection of cardiac dysfunction or myocardial damage have been used in various settings of heart failure [21]. We found that most patients with DMD or BMB had an elevated level of serum troponin $\mathrm{T}$ at baseline, probably reflecting latent and progressive myocardial damage, and that those with elevated levels of plasma BNP were more likely to have a substantial LGE. BNP or N-terminal pro-BNP is the most widely used biomarker for the detection and management of heart failure, which may be useful for risk stratification in pediatric outpatients [22] and in patients with DMD [23]. Our 
results suggest that significant elevation in plasma BNP levels may be a sign of substantial myocardial fibrosis.

\section{Study Limitations}

This study has several limitations. First, it is a single-centre retrospective study and is subject to selection bias. Although the patients were treated at the discretion of their physicians, the latter were all experienced pediatric cardiologists. Second, the small sample size did not allow for separate analysis by the type of muscular dystrophy. Although the natural course of progressive cardiac involvement might differ between DMD and BMD, cardioprotective effects of ACEI were found to be independent of the type of muscular dystrophy in multivariate analysis. Third, we did not assess the effects of combination therapy with aldosterone antagonists, which may attenuate progressive LV systolic dysfunction in patients with DMD [24] or BMD. Therefore, further studies are needed to expand upon the present findings in an effort to improve the management of muscular dystrophy patients with cardiac involvement and normal LVEF.

\section{Conclusions}

ACEI attenuated the age-related decline in LVEF only in patients with DMD or BMD and reduced LVEF. However, ACEI use did not affect the age-related increase in myocardial fibrosis in patients with DMD or BMD.

Funding: This study was funded by the Miyata Cardiac Research Promotion Foundation (to T.A.).

Conflict of Interest: The authors declare that they have no conflict of interest. 
Aikawa et al. PEDC-D-18-00579

Page 13

Ethical approval: "All procedures performed in studies involving human participants were in accordance with the ethical standards of the institutional and/or national research committee and with the 1964 Helsinki declaration and its later amendments or comparable ethical standards. For this type of study formal consent is not required.” 


\section{References}

1. Eagle M, Baudouin SV, Chandler C, Giddings DR, Bullock R, Bushby K (2002) Survival in Duchenne muscular dystrophy: improvements in life expectancy since 1967 and the impact of home nocturnal ventilation. Neuromuscul Disord 12:926-929. https://doi.org/10.1016/S0960-8966(02)00140-2

2. Melacini P, Fanin M, Danieli GA, Villanova C, Martinello F, Miorin M, Freda MP, Miorelli M, Mostacciuolo ML, Fasoli G, Angelini C, Volta SD (1996) Myocardial involvement is very frequent among patients affected with subclinical Becker's muscular dystrophy. Circulation

94:3168-3175. https://doi.org/10.1161/01.CIR.94.12.3168

3. Jefferies JL, Eidem BW, Belmont JW, Craigen WJ, Ware SM, Fernbach SD, Neish SR, Smith EO, Towbin JA (2005) Genetic predictors and remodeling of dilated cardiomyopathy in muscular dystrophy. Circulation 112:2799-2804. https://doi.org/10.1161/CIRCULATIONAHA.104.528281

4. Melacini P, Fanin M, Danieli GA, Fasoli G, Villanova C, Angelini C, Vitiello L, Miorelli M, Buja GF, Mostacciuolo ML, Pegoraro E, Volta SD (1993) Cardiac involvement in Becker muscular dystrophy. J Am Coll Cardiol 22:1927-1934. https://doi.org/10.1016/0735-1097(93)90781-U

5. Silva MC, Meira ZM, Gurgel Giannetti J, da Silva MM, Campos AF, Barbosa Mde M, Starling Filho GM, Ferreira Rde A, Zatz M, Rochitte CE (2007) Myocardial delayed enhancement by magnetic resonance imaging in patients with muscular dystrophy. J Am Coll Cardiol 49:1874-1879. https://doi.org/10.1016/j.jacc.2006.10.078

6. Tandon A, Villa CR, Hor KN, Jefferies JL, Gao Z, Towbin JA, Wong BL, Mazur W, Fleck RJ, Sticka JJ, Benson DW, Taylor MD (2015) Myocardial fibrosis burden predicts left ventricular ejection fraction and is associated with age and steroid treatment duration in duchenne muscular dystrophy. J Am Heart Assoc

4. https://doi.org/10.1161/JAHA.114.001338

7. Feingold B, Mahle WT, Auerbach S, Clemens P, Domenighetti AA, Jefferies JL, Judge DP, Lal AK, Markham LW, Parks WJ, Tsuda T, Wang PJ, Yoo SJ; American Heart Association Pediatric Heart Failure Committee of the Council on Cardiovascular Disease in the Young; Council on Clinical Cardiology; Council on Cardiovascular Radiology and Intervention; Council on Functional Genomics and Translational Biology; and Stroke Council (2017) Management of cardiac involvement associated with neuromuscular diseases: a scientific statement From the American Heart Association. Circulation 136:e200-e231. https://doi.org/10.1161/CIR.0000000000000526

8. Silva MC, Magalhaes TA, Meira ZM, Rassi CH, Andrade AC, Gutierrez PS, 
Azevedo CF, Gurgel-Giannetti J, Vainzof M, Zatz M, Kalil-Filho R, Rochitte CE (2017) Myocardial fibrosis progression in Duchenne and Becker muscular dystrophy: a randomized clinical trial. JAMA Cardiol 2:190-199. https://doi.org/10.1001/jamacardio.2016.4801

9. Duboc D, Meune C, Pierre B, Wahbi K, Eymard B, Toutain A, Berard C, Vaksmann G, Weber S, Becane HM (2007) Perindopril preventive treatment on mortality in Duchenne muscular dystrophy: 10 years' follow-up. Am Heart J 154:596-602. https://doi.org/10.1016/j.ahj.2007.05.014

10. Aikawa T, Naya M, Obara M, Oyama-Manabe N, Manabe O, Magota K, Ito YM, Katoh C, Tamaki N (2017) Regional interaction between myocardial sympathetic denervation, contractile dysfunction, and fibrosis in heart failure with preserved ejection fraction: ${ }^{11}$ C-hydroxyephedrine PET study. Eur J Nucl Med Mol Imaging 44:1897-1905. https://doi.org/10.1007/s00259-017-3760-y

11. Aikawa T, Oyama-Manabe N, Naya M, Ohira H, Sugimoto A, Tsujino I, Obara M, Manabe O, Kudo K, Tsutsui H, Tamaki N (2017) Delayed contrast-enhanced computed tomography in patients with known or suspected cardiac sarcoidosis: a feasibility study. Eur Radiol 27:4054-4063. https://doi.org/10.1007/s00330-017-4824-X

12. Nassenstein K, Breuckmann F, Bucher C, Kaiser G, Konorza T, Schafer L, Konietzka I, de Greiff A, Heusch G, Erbel R, Barkhausen J (2008) How much myocardial damage is necessary to enable detection of focal late gadolinium enhancement at cardiac MR imaging? Radiology 249:829-835. https://doi.org/10.1148/radiol.2493080457

13. Allen HD, Flanigan KM, Thrush PT, Dvorchik I, Yin H, Canter C, Connolly AM, Parrish M, McDonald CM, Braunlin E, Colan SD, Day J, Darras B, Mendell JR (2013) A randomized, double-blind trial of lisinopril and losartan for the treatment of cardiomyopathy in duchenne muscular dystrophy. PLoS Curr

5. https://doi.org/10.1371/currents.md.2cc69a1dae4be7dfe2bcb420024ea865

14. Viollet L, Thrush PT, Flanigan KM, Mendell JR, Allen HD (2012) Effects of angiotensin-converting enzyme inhibitors and/or beta blockers on the cardiomyopathy in Duchenne muscular dystrophy. Am J Cardiol 110:98-102. https://doi.org/10.1016/j.amjcard.2012.02.064

15. Duboc D, Meune C, Lerebours G, Devaux JY, Vaksmann G, Becane HM (2005) Effect of perindopril on the onset and progression of left ventricular dysfunction in Duchenne muscular dystrophy. J Am Coll Cardiol 45:855-857. https://doi.org/10.1016/j.jacc.2004.09.078

16. Schram G, Fournier A, Leduc H, Dahdah N, Therien J, Vanasse M, Khairy P (2013) All-cause mortality and cardiovascular outcomes with prophylactic steroid therapy 
in Duchenne muscular dystrophy. J Am Coll Cardiol

61:948-954. https://doi.org/10.1016/j.jacc.2012.12.008

17. Lonn EM, Yusuf S, Jha P, Montague TJ, Teo KK, Benedict CR, Pitt B (1994)

Emerging role of angiotensin-converting enzyme inhibitors in cardiac and vascular protection. Circulation 90:2056-2069. https://doi.org/10.1161/01.CIR.90.4.2056

18. Mewton N, Liu CY, Croisille P, Bluemke D, Lima JA (2011) Assessment of myocardial fibrosis with cardiovascular magnetic resonance. J Am Coll Cardiol 57:891-903. https://doi.org/10.1016/j.jacc.2010.11.013

19. Hor KN, Wansapura J, Markham LW, Mazur W, Cripe LH, Fleck R, Benson DW, Gottliebson WM (2009) Circumferential strain analysis identifies strata of cardiomyopathy in Duchenne muscular dystrophy: a cardiac magnetic resonance tagging study. J Am Coll Cardiol 53:1204-1210. https://doi.org/10.1016/j.jacc.2008.12.032

20. Soslow JH, Damon SM, Crum K, Lawson MA, Slaughter JC, Xu M, Arai AE, Sawyer DB, Parra DA, Damon BM, Markham LW (2016) Increased myocardial native $\mathrm{T} 1$ and extracellular volume in patients with Duchenne muscular dystrophy. $\mathrm{J}$ Cardiovasc Magn Reson 18:5. https://doi.org/10.1186/s12968-016-0224-7

21. Maisel AS, Bhalla V, Braunwald E (2006) Cardiac biomarkers: a contemporary status report. Nat Clin Pract Cardiovasc Med 3:24-34. https://doi.org/10.1038/ncpcardio0405

22. Price JF, Thomas AK, Grenier M, Eidem BW, O'Brian Smith E, Denfield SW, Towbin JA, Dreyer WJ (2006) B-type natriuretic peptide predicts adverse cardiovascular events in pediatric outpatients with chronic left ventricular systolic dysfunction. Circulation 114:1063-1069. https://doi.org/10.1161/circulationaha.105.608869

23. Cheeran D, Khan S, Khera R, Bhatt A, Garg S, Grodin JL, Morlend R, Araj FG, Amin AA, Thibodeau JT, Das S, Drazner MH, Mammen PPA (2017) Predictors of death in adults with Duchenne muscular dystrophy-associated cardiomyopathy. J Am Heart Assoc 6. https://doi.org/10.1161/jaha.117.006340

24. Raman SV, Hor KN, Mazur W, Halnon NJ, Kissel JT, He X, Tran T, Smart S, McCarthy B, Taylor MD, Jefferies JL, Rafael-Fortney JA, Lowe J, Roble SL, Cripe LH (2015) Eplerenone for early cardiomyopathy in Duchenne muscular dystrophy: a randomised, double-blind, placebo-controlled trial. Lancet Neurol 14:153-161. https://doi.org/10.1016/s1474-4422(14)70318-7 


\section{Figure legends}

Fig. 1 Representative late gadolinium enhancement images of a 15-year-old boy with

Becker muscular dystrophy. (a) Hyperenhanced myocardium was seen in the inferior to lateral and septal wall. (b) Yellow areas denote myocardium $\geq 5$ standard deviations above the mean for signal intensity in the remote myocardium
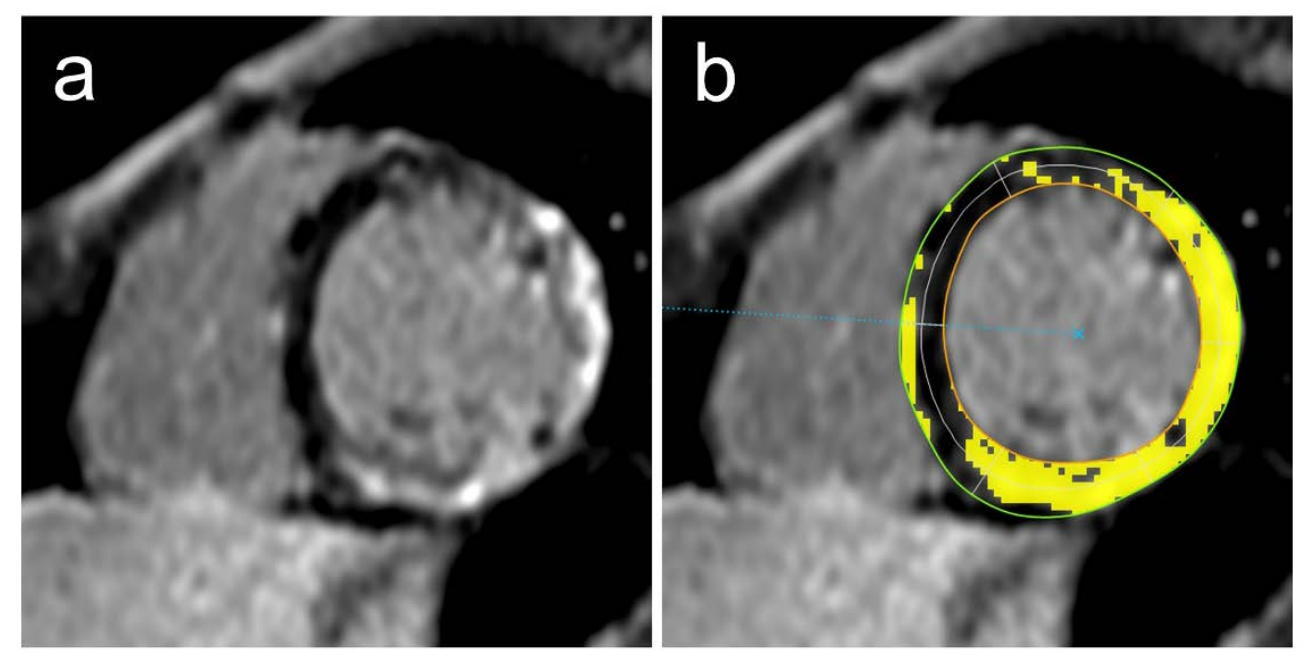

Fig. 2 Correlation between the extent of late gadolinium enhancement (LGE) and left ventricular ejection fraction (LVEF) in patients with Duchenne muscular dystrophy (DMD, red circles) and Becker muscular dystrophy (BMD, blue circles). *One patient with DMD did not undergo LGE imaging at the first CMR

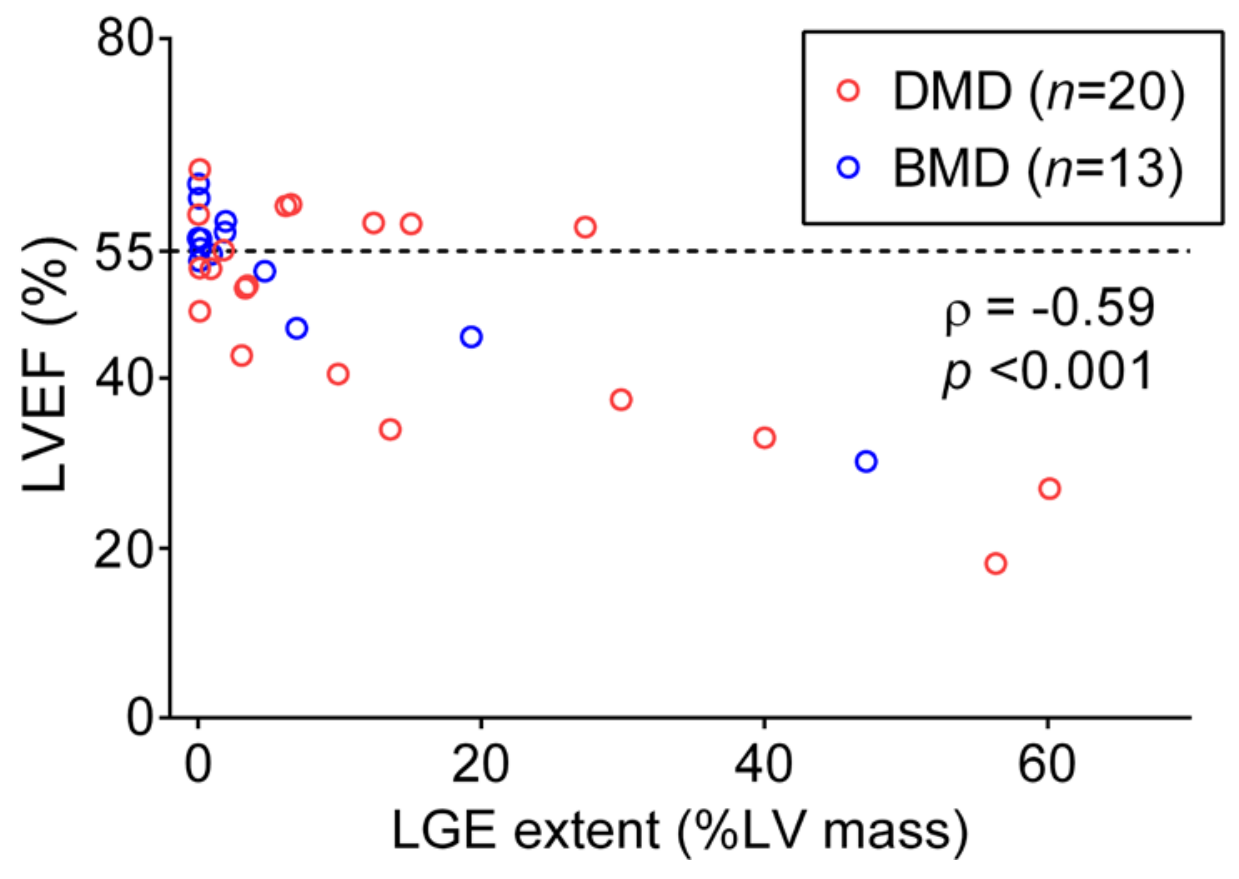


Fig. 3 Longitudinal relationship between age and left ventricular ejection fraction (LVEF).

Each patient (red circles, Duchenne muscular dystrophy [DMD]; blue circles, Becker muscular dystrophy [BMD]) is connected by a colored line during follow-up. The filled and open circles denote patients treated with and without angiotensin-converting enzyme inhibitor (ACEI), respectively. LVEF decreased by $0.65 \% \pm 0.31 \%$ ( $\beta \pm$ standard error) per year in a mixed-effects model adjusted for age only

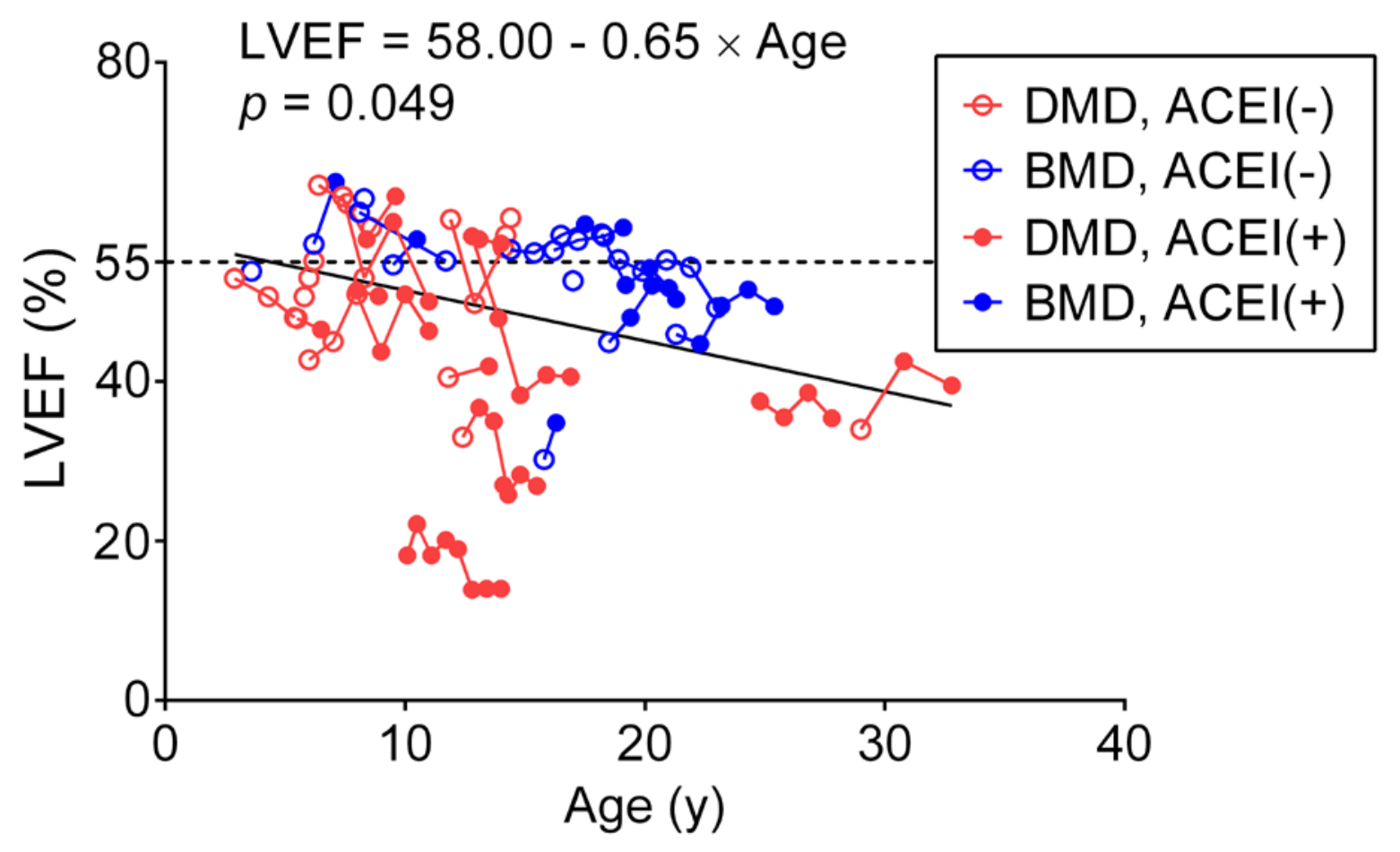


Table 1. Patients characteristics at the first cardiovascular magnetic resonance study

\begin{tabular}{|c|c|c|c|}
\hline Variable & $\begin{array}{c}\text { DMD } \\
(n=21)\end{array}$ & $\begin{array}{c}\text { BMD } \\
(n=13)\end{array}$ & $\boldsymbol{p}$ \\
\hline Age (y) & $10.1(6.1-13.6)$ & $15.8(8.2-18.4)$ & 0.08 \\
\hline Male & $20(95)$ & $13(100)$ & 1.00 \\
\hline Body mass index $\left(\mathrm{kg} / \mathrm{m}^{2}\right)$ & $16.6(15.2-21.3)$ & $18.0(14.7-19.4)$ & 1.00 \\
\hline Wheelchair bound & $8(38)$ & $0(0)$ & 0.013 \\
\hline \multicolumn{4}{|l|}{ Medications } \\
\hline ACEI & $5(24)$ & $0(0)$ & 0.13 \\
\hline Start at LVEF $\geq 55 \%$ & $2(10)$ & $0(0)$ & 0.51 \\
\hline Start at LVEF <55\% & $3(14)$ & $0(0)$ & 0.27 \\
\hline Duration of ACEI use (y) & $1.6(0.8-2.3)$ & - & - \\
\hline Angiotensin II receptor blocker & $0(0)$ & $0(0)$ & 1.00 \\
\hline$\beta$-blocker & $3(14)$ & $0(0)$ & 0.27 \\
\hline Duration of $\beta$-blocker use (y) & $1.3(0.8-2.3)$ & - & - \\
\hline Steroid & $8(38)$ & $0(0)$ & 0.013 \\
\hline Duration of steroid use (y) & $1.0(0.5-3.0)$ & - & - \\
\hline \multicolumn{4}{|l|}{ Blood data* } \\
\hline Total creatine kinase (IU/L) & 8842 (2845-13888) & 2713 (1336-12961) & 0.034 \\
\hline Brain natriuretic peptide (pg/mL) & $15.2(6.4-24.6)$ & $5.8(5.8-7.3)$ & 0.014 \\
\hline $\begin{array}{l}\text { Human atrial natriuretic peptide }(\mathrm{pg} / \mathrm{mL} \text {, } \\
n=32)\end{array}$ & $23.3(17.7-33.0)$ & $22.9(12.2-30.6)$ & 0.36 \\
\hline Troponin T (pg/mL, $n=31)$ & $0.085(0.056-0.122)$ & $0.015(0.009-0.028)$ & $<0.001$ \\
\hline \multicolumn{4}{|l|}{ CMR findings } \\
\hline LVEF (\%) & $52.9(39.0-58.8)$ & $55.3(49.3-57.9)$ & 0.50 \\
\hline LV end-diastolic volume index (mL/m²) & $57.5(47.6-78.0)$ & 68.9(46.8-72.3) & 0.72 \\
\hline LV end-systolic volume index $\left(\mathrm{mL} / \mathrm{m}^{2}\right)$ & $26.0(21.8-44.7)$ & $30.8(19.7-37.9)$ & 1.00 \\
\hline LV mass index $\left(\mathrm{g} / \mathrm{m}^{2}\right)$ & $47.7(42.8-50.8)$ & $48.2(40.9-55.8)$ & 0.92 \\
\hline LGE extent (\% of total LV mass, $n=33$ ) $\dagger$ & $6.4(1.1-24.3)$ & $1.0(0.1-5.8)$ & 0.07 \\
\hline Patients undergoing $\geq 2$ CMR study & $14(67)$ & $10(77)$ & 0.70 \\
\hline
\end{tabular}

Values are median (interquartile range) or $\mathrm{n}(\%)$. LV analyses were normalized by body surface area $\left(\mathrm{m}^{2}\right)$.

*Normal reference values for brain natriuretic peptide, human atrial natriuretic peptide, and troponin T were $\leq 18.4 \mathrm{pg} / \mathrm{mL}, \leq 43.0 \mathrm{pg} / \mathrm{ml}$, and $\leq 0.014 \mathrm{ng} / \mathrm{mL}$, respectively.

†One patient with DMD did not undergo LGE imaging at the first CMR.

ACEI: angiotensin converting enzyme inhibitor; BMD: Becker muscular dystrophy; CMR: cardiovascular magnetic resonance; DMD: Duchenne muscular dystrophy; LGE: late gadolinium enhancement; LV: left ventricular; LVEF: left ventricular ejection fraction. 
Table 2. Patients characteristics at the last cardiovascular magnetic resonance study $(n=24)$

\begin{tabular}{|c|c|c|c|}
\hline Variable & $\begin{array}{l}\text { DMD } \\
(n=14)\end{array}$ & $\begin{array}{c}\text { BMD } \\
(n=10)\end{array}$ & $\boldsymbol{p}$ \\
\hline Follow-up duration (y) & $3.0(2.1-3.6)$ & $2.9(1.0-3.7)$ & 0.88 \\
\hline Age $(y)$ & $13.8(9.4-15.9)$ & $18.3(11.4-21.7)$ & 0.15 \\
\hline Male & $13(93)$ & $10(100)$ & 1.00 \\
\hline Body mass index $\left(\mathrm{kg} / \mathrm{m}^{2}\right)$ & $16.5(14.0-19.1)$ & $19.0(16.2-22.6)$ & 0.15 \\
\hline Wheelchair-bound & $8(57)$ & $0(0)$ & 0.006 \\
\hline \multicolumn{4}{|l|}{ Medications } \\
\hline ACEI & $12(86)$ & $8(80)$ & 1.00 \\
\hline Start at LVEF $\geq 55 \%$ & $3(21)$ & $4(40)$ & 0.39 \\
\hline Start at LVEF < $55 \%$ & $9(64)$ & $4(40)$ & 0.41 \\
\hline Duration of ACEI use (y) & $2.7(1.5-4.2)$ & $1.0(0.6-2.7)$ & 0.049 \\
\hline Angiotensin II receptor blocker & $1(7)$ & $0(0)$ & 1.00 \\
\hline $\begin{array}{l}\text { Duration of angiotensin II receptor } \\
\text { blocker use (y) }\end{array}$ & $1.0(1.0-1.0)$ & - & - \\
\hline$\beta$-blocker & $5(36)$ & $0(0)$ & 0.053 \\
\hline Duration of $\beta$-blocker use (y) & $2.8(1.1-5.0)$ & - & - \\
\hline Steroid & $5(36)$ & $0(0)$ & 0.053 \\
\hline Duration of steroid use (y) & $3.7(3.0-5.2)$ & - & - \\
\hline \multicolumn{4}{|l|}{ Blood data* } \\
\hline Total creatine kinase (IU/L, $n=23$ ) & 4374 (1308-9122) & 2012 (692-7684) & 0.28 \\
\hline Brain natriuretic peptide (pg/mL, $n=23$ ) & $9.2(5.8-36.5)$ & $5.8(5.8-16.8)$ & 0.41 \\
\hline $\begin{array}{l}\text { Human atrial natriuretic peptide }(\mathrm{pg} / \mathrm{mL} \text {, } \\
n=21)\end{array}$ & $27.2(19.2-55.9)$ & $12.5(10.4-20.5)$ & 0.008 \\
\hline Troponin T (pg/mL, $n=20)$ & $0.080(0.035-0.115)$ & $0.012(0.011-0.037)$ & 0.003 \\
\hline \multicolumn{4}{|l|}{ CMR findings } \\
\hline LVEF (\%) & $46.4(38.5-52.2)$ & $53.4(49.4-59.4)$ & 0.043 \\
\hline LV end-diastolic volume index $\left(\mathrm{mL} / \mathrm{m}^{2}\right)$ & $58.8(48.5-76.6)$ & $59.2(47.8-82.5)$ & 0.98 \\
\hline $\mathrm{LV}$ end-systolic volume index $\left(\mathrm{mL} / \mathrm{m}^{2}\right)$ & $31.2(23.5-47.4)$ & $29.8(19.4-35.6)$ & 0.50 \\
\hline LV mass index $\left(\mathrm{g} / \mathrm{m}^{2}\right)$ & $47.5(44.9-60.3)$ & $47.4(42.9-55.1)$ & 0.66 \\
\hline LGE extent (\% of total LV mass) & $11.6(7.6-36.2)$ & $1.5(0.3-8.4)$ & 0.018 \\
\hline
\end{tabular}

Values are median (interquartile range) or $\mathrm{n}(\%)$. LV analyses were normalized by body surface area $\left(\mathrm{m}^{2}\right)$.

*Normal reference values for brain natriuretic peptide, human atrial natriuretic peptide, and troponin T were $\leq 18.4 \mathrm{pg} / \mathrm{mL}, \leq 43.0 \mathrm{pg} / \mathrm{ml}$, and $\leq 0.014 \mathrm{ng} / \mathrm{mL}$, respectively.

ACEI: angiotensin converting enzyme inhibitor; BMD: Becker muscular dystrophy; CMR: cardiovascular magnetic resonance; DMD: Duchenne muscular dystrophy; LGE: late gadolinium enhancement; LV: left ventricular; LVEF: left ventricular ejection fraction. 
Table 3. Mixed-effects model to identify predictors of left ventricular ejection fraction (LVEF, \%)

\begin{tabular}{|c|c|c|c|c|c|c|}
\hline & \multicolumn{3}{|c|}{ Model 1} & \multicolumn{3}{|c|}{ Model 2} \\
\hline & $\boldsymbol{\beta}$ & $95 \% \mathrm{CI}$ & $p$ & $\beta$ & $95 \% \mathrm{CI}$ & $p$ \\
\hline Intercept & 61.1 & 52.3 to 70.0 & $<0.001$ & 61.5 & 52.5 to 70.4 & $<0.001$ \\
\hline Age (y) & -1.1 & -1.8 to -0.4 & 0.005 & -1.1 & -1.8 to -0.4 & 0.005 \\
\hline BMD & 5.6 & -3.6 to 14.8 & 0.22 & 5.3 & -4.1 to 14.7 & 0.26 \\
\hline Steroid use & -4.5 & -11.2 to 2.2 & 0.19 & -5.0 & -11.9 to 1.9 & 0.15 \\
\hline ACEI use & 3.1 & 0.8 to 5.4 & 0.009 & - & - & - \\
\hline Start at $\mathrm{LVEF} \geq 55 \%$ & - & - & - & 2.2 & -1.5 to 5.8 & 0.24 \\
\hline Start at LVEF < $55 \%$ & - & - & - & 3.7 & 0.9 to 6.4 & 0.010 \\
\hline
\end{tabular}

ACEI: angiotensin converting enzyme inhibitor; BMD: Becker muscular dystrophy; CI: confidence interval 
Table 4. Mixed-effects model to identify predictors of the extent of late gadolinium enhancement (LGE, \% of left ventricular mass)

\begin{tabular}{|c|c|c|c|c|c|c|c|c|c|}
\hline & \multicolumn{3}{|c|}{ Model 1} & \multicolumn{3}{|c|}{ Model 2} & \multicolumn{3}{|c|}{ Model 3} \\
\hline & $\beta$ & 95\% CI & $p$ & $\boldsymbol{\beta}$ & 95\% CI & $p$ & $\beta$ & $95 \% \mathrm{CI}$ & $p$ \\
\hline Intercept & -0.2 & $\begin{array}{c}-12.3 \text { to } \\
11.8\end{array}$ & 0.97 & -0.7 & $\begin{array}{c}-13.2 \text { to } \\
11.7\end{array}$ & 0.90 & -0.8 & $\begin{array}{c}-13.2 \text { to } \\
11.7\end{array}$ & 0.90 \\
\hline Age ( & 1.5 & 0.5 to 2.4 & 0.005 & 1.5 & 0.5 to 2.6 & 0.006 & 1.5 & 0.5 to 2.6 & 0.006 \\
\hline $\mathrm{BM}$ & -10.7 & -22.8 to 1.3 & $0.0 \varepsilon$ & -11.1 & -23.4 to 1.2 & $0 .($ & -11.2 & -23.5 to 1.2 & 0.07 \\
\hline ACEI use & - & - & - & -0.5 & -3.5 to 2.4 & 0.71 & - & - & - \\
\hline $\begin{array}{l}\text { Start at LVEF } \\
\geq 55 \%\end{array}$ & - & - & - & - & - & - & -1.5 & -6.4 to 3.4 & 0.55 \\
\hline $\begin{array}{l}\text { Start at LVEF } \\
<55 \%\end{array}$ & - & - & - & - & - & - & -0.1 & -3.6 to 3.5 & 0.97 \\
\hline
\end{tabular}

ACEI: angiotensin converting enzyme inhibitor; BMD: Becker muscular dystrophy; CI: confidence interval; LGE: late gadolinium enhancement; LVEF: left ventricular ejection fraction. 